\title{
Géolinguistique
}

14 | 2013

Varia

\section{Hydrotoponymes du massif de la Chartreuse : essai d'inventaire}

Hydrotoponyms of the Chartreuse Massif: Inventory Test

Jeanine Élisa Médélice

\section{Q OpenEdition}

\section{Journals}

Édition électronique

URL : http://journals.openedition.org/geolinguistique/793

DOI : 10.4000/geolinguistique.793

ISSN : 2650-8176

Éditeur

UGA Éditions/Université Grenoble Alpes

\section{Édition imprimée}

Date de publication : 15 décembre 2013

Pagination : 21-34

ISBN : 978-2-84310-264-6

ISSN : 0761-9081

Référence électronique

Jeanine Élisa Médélice, « Hydrotoponymes du massif de la Chartreuse : essai d'inventaire »,

Géolinguistique [En ligne], 14 | 2013, mis en ligne le 30 avril 2019, consulté le 06 novembre 2020. URL http://journals.openedition.org/geolinguistique/793; DOI : https://doi.org/10.4000/geolinguistique. 793 


\title{
Hydrotoponymes du massif de la Chartreuse : essai d'inventaire ${ }^{1}$
}

\author{
Jeanine Élisa Médélice \\ Université Grenoble Alpes, Gipsa-lab (UMR 5216)
}

\section{Résumé}

Cet article présente et évalue les possibilités qu'offre le domaine spécifique des hydrotoponymes (microtoponymes liés à la présence de l'eau) dans la perspective de l' «Inventaire microtoponymique du massif de la Chartreuse». Ce projet a été initié par le Centre de dialectologie (GIPSA-Lab, équipe Systèmes linguistiques et Dialectologie, UMR 5216) avec le soutien de la région Rhône-Alpes.

Le classement adopté est hiérarchisé de la façon suivante : deux parties (référence directe à l'eau / référence indirecte à l'eau) à l'intérieur desquelles les motivations, mots, bases... sont présentés sous forme de listes : pour donner un exemple, sous l'entrée «source»sont réunies les diverses données (source / font / fontaine...) avec leur origine et accompagnées de quelques exemples. Ces listes ne sont pas exhaustives, le but de cette présentation se limitant à une évaluation.

\section{Mots-clés}

Toponymie, géolinguistique, Chartreuse.

1. À partir d'une communication réalisée le 13 mars 2013 pour la journée d'études «Valorisation de la recherche en microtoponymie», dans le cadre du projet «Inventaire microtoponymique du massif de la Chartreuse » de l'ARC 5 de la région Rhône-Alpes. 


\begin{abstract}
This paper presents and evaluates the potential of the specific area of "hydrotoponyms" (microtoponyms related to the presence of water) in the context of the "Inventaire microtoponymique du massif de la Chartreuse". This project was initiated by the Centre de dialectologie (GIPSA-Lab, équipe Systèmes linguistiques et Dialectologie, UMR 5216) with the support of the Rhône-Alpes Region.

The hierarchical classification adopted is as follows: two parts (direct reference to water / indirect reference to water) within which the motives, words, bases... are presented in the form of lists: to give an example, under the entry "source" gathered various data (source / font / fontaine...) with their origin and accompanied by a few examples. These lists are not thorough; the purpose of this presentation is limited to an evaluation.
\end{abstract}

\title{
Keywords
}

Toponymy, Geolinguistics, Chartreuse.

Il existe un lien évident entre les cours d'eau, les lieux qu'ils traversent et les habitats qui les bordent. La référence aux hydronymes est fréquente dans la désignation des lieux : la localité Entre-deux-Guiers, qui s'est développée au confluent du Guiers-vif et du Guiers-mort, en est une parfaite illustration ainsi que son vis-à-vis Les Échelles où sont relevés les toponymes : Dans Guiers et Contre le Guiers. Il en va de même pour le nom des voies de communication (odonyme) menant vers un cours d'eau ou vers un élément lié à l'eau. Au Fontanil, localité au nom motivé par la présence de sources et proche de la rivière, on traverse la Rue de l'Étang, Rue du Drac...

La présence de l'eau se signale, en toponymie, de façon directe, comme on vient de le voir ci-dessus, ou indirecte lorsque le toponyme signale la présence de végétaux, d'animaux et/ou d'activités humaines requérant la présence de l'eau.

Dans les zones qui ont vu se développer une forte densité immobilière, le nom des anciennes parcelles survit souvent dans le nom des rues (ou des chemins).

La richesse (la densité) hydronymique du massif de la Chartreuse justifie l'intérêt d'une étude portant sur les hydrotoponymes : c'est-à-dire les noms de lieux qui trouvent leur motivation dans la présence de l'eau. Ce qui suit en est un aperçu. 
Le signalement direct se traduit par l'utilisation de noms comme :

1.1. «Eau»: les continuateurs du latin AQUA et de ses dérivés, nombreux et variés en domaine gallo-roman, sont peu présents en Chartreuse. Ils se rencontrent cependant dans quelques toponymes, associés à un adjectif : Aigue Sort (Saint-Pierre-d'Entremont-Savoie), Aiguenoire (hameau de Entre-deux-Guiers / étang à Voiron), Aigue Belle (Saint-Pancrasse) et Aiguebelette ainsi que Eygala, du latin *aqualem, qui désigne un ruisseau (La Buisse / Voreppe) ou un hameau (Corenc / Meylan). L'utilisation, sous forme suffixée, pour nommer un faible cours d'eau est extrêmement fréquente. Pilot de Thorey mentionne Aiguebelle, une fontaine à Saint-Pancrasse.

1.2. «Sources» : nombreuses sont les références aux sources.

1.2.1. Du latin FONS, -TIS, acc. FONTEm 'source' / et son dérivé FONTANA 'source captée, fontaine' (carte 1).

Aucun toponyme ne se présente aujourd'hui sous la forme simple, pourtant attestée à plusieurs reprises dans les textes anciens. Certaines «Fontaine» modernes ont pour première attestation un «fons $+»$ : la première mention parvenue jusqu’à nous de La Fontaine (Saint-Étienne-deCrossey) est «Fons de Monferra» au XIV siècle (Pilot de Thorey, p. 151). D'autres, toujours mentionnées par Pilot de Thorey, n'apparaissent sur aucun cadastre. Cela peut s'expliquer par la proximité de «fond» issu de FUNDU 'fond'. Certains «fond» sont, en Chartreuse comme ailleurs, des réinterprétations de «font». Ils ne sont pris en compte que lorsqu'une forme ancienne atteste de la réinterprétation. Cependant face à l'hydronyme Fonds des Chartreux ou l'odonyme Route de Très Fonds (qui devient Chemin de Tréfonds au bout de quelques centaines de mètres, à Voreppe), le besoin d'attestation semble un peu superflu.

Font apparaît aussi dans quelques formes composées : Fontvielle (Quaix); Bellefont, à l'extrémité occidentale de la barre de la Dent de Crolles: Rochers (dôme/col) de Bellefont. À noter que Bellefont se rencontre fréquemment orthographié «Bellefond» (sur certains panneaux de circuit de randonnée). La rue et le ruisseau de Fontabert (La Buisse) témoigne de l'existence passée d'une source (ou d'une fontaine) couverte.

Les microtoponymes issus de FONTANA 'source captée' (et/ou francisés plus tard) sont très nombreux. En l'absence de datation précise, il est difficile de faire la part des «source» et des «fontaine» au sens que leur donne le français. 


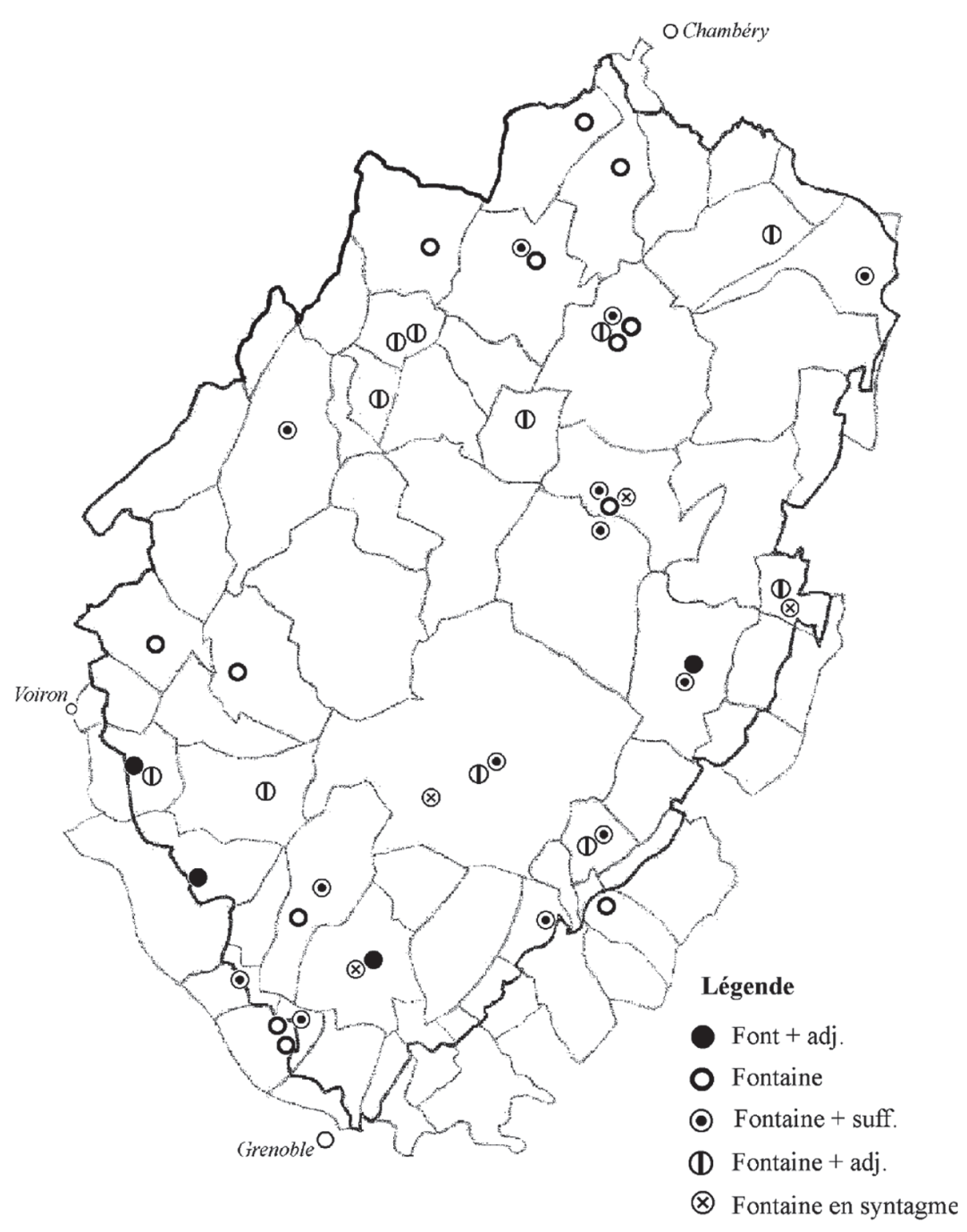

Carte 1. - Les continuateurs de FONTEm. 
Les formes sont variées : au singulier ou au pluriel, en syntagme, avec ou sans l'article, suivies d'un adjectif (ou précédées, dans le cas de l'adjectif numéral). Certaines communes en connaissent plusieurs : Fontaine (Saint-Joseph-de-Rivière); La Fontaine (Saint-Cassin / Entremont-le-Vieux / Le Fontanil / Saint-Pierre-d'Entremont-Isère / SaintThibaud-de-Couz); Les Fontaines (Bernin / Entremont-le-Vieux / Le Fontanil / Saint-Pancrasse / Attignat-Oncin / Vimines).

Avec un adjectif : Fontaine marmeleuse (Entremont-le-Vieux); La Fontaine froide (Corbel); Fontaine Froide (relief, Saint-Pancrasse / Saint-Vincent-de-Mercuze); Fontaine Romaine (La Buisse); Les Trois Fontaines (La Placette); Fontaine Quint (Saint-Pierre-de-Chartreuse); Neuf-fontaines (Apremont); Fontaine saine, Fontaine entier (La Bauche); Fontaine Entier (Saint-Pierre-de-Genebroz).

En syntagme : Fontaine du Nays (Quaix); Fontaine de l'Oursière (Saint-Pierre-de-Chartreuse); Sur la Fontaine (Saint-Pierre-d'Entremont-Savoie); Fontaine du Fou (Saint-Vincent-de-Mercuze).

Nombreux aussi sont les microtoponymes issus d'une suffixation à partir de Fontana : Le Fontanil; Le Fontanil (Miribel-les-Échelles / Saint-Pierre-de-Chartreuse); Fontanieu / Fontanieux (Saint-Pierred'Entremont-Savoie); Fontanieux (Saint-Pierre-d'Entremont-Isère) Les Fontanieux (Saint-Thibaud-de-Couz); Les Fontanettes (Lumbin / Les Marches / Saint-Pancrasse); Fontenette (Saint-Pierre-d'EntremontIsère); Les Fontenettes (Proveysieux), anciennement «Fontanettes»; Aux Fontenettes (Saint-Bernard-du-Touvet); Fontanette (Entremontle-Vieux). Les odonymes qui en signalent la direction sont aussi en nombre important: Chemin des trois Fontaines (Pommiers-La Placette); Allée de la Fontaine aux Merles (Voreppe); Chemin des Fontaines (Bernin); Place de la Fontaine (Crolles)...

1.2.2. Les microtoponymes faisant apparaître «Source» sont plus rares; Sources (Saint-Étienne-de-Crossey) et La source du Guiers (Saint-Pierre-de-Chartreuse) font figure d'exception. Les références se limitent à quelques rues et chemins : rue des Sources (Crolles); chemin de la Source (Saint-Vincent-de-Mercuze). Pourtant, sur la carte de l'ALJA (nº 136 «Source»), les relevés attestent majoritairement de formes de même origine que le français : fpr. ['sorsa].

1.2.3. Duy (du latin DUCE 'guide, qui conduit'), avec des graphies variées et au féminin, désigne souvent la source : La Duy (Saint-Pierrede-Chartreuse). La carte «Source» de l'ALJA (déjà citée) relève [dwei] pour La Ruchère. 
1.2.4. L'idée de «source» peut s'exprimer par «goutte», notamment lorsqu'elle est à l'origine d'un ruisseau à faible débit : Goutte (Les Marches); Les Dégouttes (Barraux). «Goutte» peut aussi désigner un ruisseau.

1.3. «Cours d'eau».

\subsection{1. «Ruisseau».}

Sur les cartes, les petits ruisseaux portent souvent le nom du lieu qu'ils traversent; sur place, ils sont appelés «le ruisseau».

Le latin RIVU 'ruisseau' est continué par les formes [rjø], [rif], [rui] en francoprovençal (voir ALJA, carte ${ }^{\circ} 137$ «ruisseau»). Il est à l'origine de Rieux (Les Marches); Les Rieux (Saint-Pierre-d'Entremont-Isère); plus souvent de voies de communication: Chemin des Rieux (Biviers); chemin Rieux (Saint-Hilaire); Rue du Rif Tronchard (Le Fontanil). Dans la désignation de ruisseaux, l'oubli du patois peut être à l'origine de pléonasme : ruisseau du Rif Mort, ruisseau du Rif Tronchard...

Le gaulois nanto désignait, au départ, une 'vallée où coule un cours d'eau'; il se rencontre fréquemment dans la désignation de cours d'eau de moindre importance ainsi que de hameaux (ou lieux-dits) qui les bordent : Au Nan / Le Nan (Saint-Pierre-d'Entremont-Isère); Nant SaintMichel (Saint-Bernard); Sur le Nant / Côte du Nant / Valet du Nant (Entremont-le-Vieux); Le Nant (ruisseau, Saint-Pierre-d'Entremont). La forme peut être suffixée : Le Nantet (Apremont / Entremont-le-Vieux); Route du Nantin (Saint-Cassin); Le Nancey (ruisseau, Chapareillan); Les Nances / Le Nantet (Apremont).

Il est à noter qu'en domaine gallo-roman, seule l'aire francoprovençale conserve partiellement un continuateur utilisé comme nom commun (carte 2). Plusieurs cours d'eau en témoignent en Savoie : Le Nant bruyant (La Motte-Servolex); Le Nan (affluent de l'Herbetant à SaintPierre-d'Entremont); le ruisseau du Nant clair, le Nant Bernard et le Nant de la Georgat sur la commune de Saint-Cassin.

1.3.2. «Rivière»: peu représenté en Chartreuse à l'exception de Fond de Rivière / En Rivière / Le Plan de Rivière (Saint-Joseph-de-Rivière).

1.3.3. «Cascade»: nombreux sont les «chemin (ou rue) de la cascade» : Traverse cascade (La Buisse); Chemin cascade (Saint-Laurentdu-Pont); peu de hameaux, autre que La Cascade (Saint-Cassin). La cascade peut être désignée, en outre, par le suivant. 


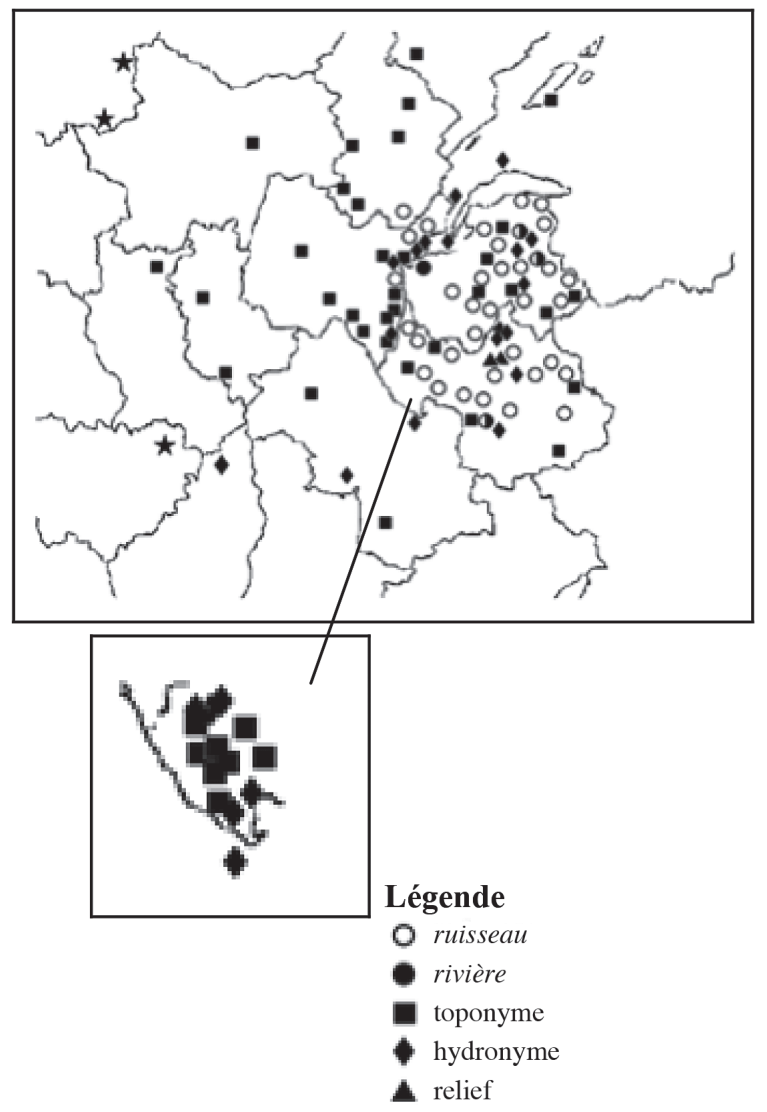

Carte 2. - Les continuateurs de *nanto, en domaine francoprovençal.

1.3.4. «Pisse» désigne des filets d'eau divers, souvent de petits ruisseaux.

Pisserotte (cascade, Saint-Julien-de-Ratz); Pissot (lieu-dit, Saint-Pierrede-Chartreuse).

\section{4. «Terrain gorgé d'eau».}

La référence à un «terrain gorgé d'eau», peu propice à la culture, est fréquente, qu'il s'agisse d'un simple terrain marneux propre à conserver l'humidité ou d'un véritable marécage (ou considéré comme tel). On rencontre une grande variété de formes, souvent construites sur des bases rencontrées fréquemment (y compris hors domaine francoprovençal). Face à des formes clairement identifiées, car issues de mots latins productifs comme FLACCIA > Flachère, on rencontre des formes 
construites sur des bases pas toujours interprétables si ce n'est qu'elles peuvent avoir une origine phonosymbolique.

1.4.1. Flachère (du lat. FLACCIA 'terrain marécageux') : La Flachère; Le Flachet (ruisseau, Les Échelles); Flacheray (Apremont).

1.4.2. Sagnes (peut-être d'origine gauloise; cf. occ. sanha) : La Sagne (Entre-deux-Guiers / Proveysieux / Miribel); Sagnes / Pré des Sagnes (Saint-Hilaire); Les Sagnes (Le Sappey / Saint-Pierre-de-Chartreuse / Saint-Pierre-d'Entremont-Isère); Les Sagnes (ruisseau et hameau, Proveysieux).

1.4.3.1. Palud (du lat. PALUdEm 'marais') : La Palud (Chapareillan / La Tronche); Paluette (Attignat-Oncin); Le Paluel (ruiseau, Voreppe / Le Fontanil); Champalud (La Buissière).

1.4.3.2. Marais (fcq *marisk) : Les Marais (Les Échelles); Le Marais (Entre-deux-Guiers); Marais (Saint-Étienne-de-Crossey); Le Marais du Chêne (Les Marches); Le Marais des Blanchisseries (Voiron); Chemin Marais (Voiron).

1.4.4.1. Fange: Les Fanges (bois, Saint-Pierre-de-Chartreuse); Le Fangeat (Voiron / Saint-Pierre-d'Entremont-Savoie / bois, Proveysieux / ruisseau, Saint-Hilaire et Saint-Pancrasse); Rue du Fangeat (Voreppe).

1.4.4.2. «Bourb-» (du gaul. *borba 'boue', fréquent dans tout le domaine gallo-roman) : La Bourbonnière (Attignat-Oncin); Borbollion (Attignat / La Bauche); Le Bourbouillon (ruisseau, Miribel-les-Échelles).

1.4.4.3. «Solliet» : d'origine obscure, solliet (sollier, sollière [féminin]) désigne le «bourbier». Il est très fréquent en francoprovençal : La Sollière (Miribel-les-Échelles); Soulliet (Saint-Pierre-d'Entremont); Les Soulliets (Saint-Laurent-du-Pont).

1.4.4.4. «Gouil-» : Le Gouillat (Le Sappey); Les Gouilles (Corbel). Outre la désignation des lieux-dits au sol humide, la base gouil- peut se retrouver pour nommer un petit cours d'eau : Le Gouillat (ruisseau, Proveysieux), une «mare» (voir ALJA, carte $n^{\circ} 141$ ), la vase (voir ALJA, carte $\mathrm{n}^{\circ} 142$ ).

1.4.4.5. «Mouill-» : La Mouilla (Saint-Vincent-de-Mercuze); Au Molliat (Saint-Pierre-d'Entremont-Savoie).

On note ici l'absence de continuateurs du latin populaire *limonem 'terre déposée par les eaux', qui signale, au contraire, un terrain riche. 
1.5. «Eau fermée»: Quelle que soit leur taille, «lac», «étang» ou «mare», les eaux fermées présentent un intérêt d'exploitation véritable. En Chartreuse, on ne relève que deux lieux-dits : L'Étang-Dauphin (Saint-Étienne-de-Crossey), Les Étangs (Entre-deux-Guiers) et quelques odonymes : Chemin de l'Étang (Saint-Étienne-de-Crossey); Chemin Étang Carré (La Buisse); Chemin de l'Étang (Le Fontanil); Route des Étangs (Voreppe); Pont de l'Étang (Chapareillan / Les Marches). Les «lac» sont peu représentés aussi : Le Lac Broz (Les Marches); Le Lac (Saint-Julien-en-Ratz); Le lac de Bey / Le lac de la Puce (Chapareillan); Rue du Lac (Le Fontanil).

1.6. «Confluent» : l'idée de «confluent» s'exprime fréquemment à l'aide des continuateurs du latin CONFLUENTE ou du gaulois condat. Les deux sont absents en Chartreuse. Seule représentante de cette motivation, une forme plus moderne : Entre-deux-Guiers, qui nomme la ville située au confluent du Guiers-mort et du Guiers-vif. Il s'agit d'une forme intéressante faisant appel aux noms même des hydronymes quand, habituellement, on rencontre une forme moins précise Entraigues; ce type est présent dans tout le domaine gallo-roman.

Lui faisant face, côté savoyard, sur la commune Les Échelles, les lieuxdits : Les Deux ruisseaux et Entre-les-deux-ruisseaux.

1.7. «Île» désigne souvent un quartier proche d'un cours d'eau et propice aux inondations.

Avenue de l'île Brune / Rue des Îles (Le Fontanil). Voreppe connaît de nombreux odonymes y faisant référence : Chemin de l'île Magnin I Chemin de l'île du Pont / Chemin de l'île Plançon, de même que Crolles qui compte huit attestations; Chemin des Îles de Coise (Chapareillan).

2. Éléments directement liés à l'eau.

Lorsqu'ils servent à traverser les rivières, ils peuvent être :

2.1. Naturels : gué (lat. vadum) : Le Gué des Planches (Attignat-Oncin); Le Gua (Proveysieux); Au Gaz (Saint-Pierre-d'Entremont-Isère); Le $\mathrm{Gaz}$ (Apremont), qui désigne ailleurs un ruisseau intermittent (Le Planolet). Un Ruisseau du Gaz coule sur la commune de Corbel.

\subsection{Construits : pont.}

«Pont» (du latin PONS/PONTE) est très fréquent, généralement accompagné d'un attributif dans la désignation de hameaux ou de lieux-dits proches d'un pont : Pont Jean Lioud (Entre-deux-Guiers); Pont StMartin (Saint-Christophe); Pont de l'Oulle (Proveysieux); Le Pont de 
May (Saint-Joseph-de-Rivière); Pont Saint Charles (Saint-Cassin); Pont du Cernon / Pont Royal (Chapareillan). Pont de l'Étang (Chapareillan / Les Marches) désigne à la fois un pont et le moulin qui le borde. Le Pont Pérant est un pont en ruine sur le Guiers-mort dans la commune de Saint-Laurent-du-Pont. Plus rarement, se rencontrent des formes suffixées : Le Pontet (Saint-Cassin / Saint-Pierre-d'Entremont); $A u$ Pontet (Proveysieux) ou accompagnées d'un adjectif : Pont neuf / Pont Vieux, deux hameaux de Cognin, cités par Vernier, dont ne survit aujourd'hui qu'une rue.

NB : aucun continuateur de gaulois *briva: Brive.

3. Éléments liés aux activités engendrées par la présence de l'eau.

Les cours d'eaux et les lacs engendraient des activités de pêche et de batelage souvent révolues aujourd'hui, qui nécessitaient une infrastructure dont témoigne la toponymie :

3.1. Port : Le Port (Aiguebelette); Rue du Port (Le Fontanil / Voreppe).

3.2. Digue : La Digue (Entre-deux-Guiers); Rue de la Digue (Les Échelles / Entre-deux-Guiers); Chemin des Digues (Voreppe).

3.3. «Écluse»: Chemin de l'Écluse (Le Fontanil); à noter que «écluse» peut être issu d'une remotivation de «cluse».

3.4. «Canal » : Les Traversières; Impasse Canal (Entre-deux-Guiers); Le Béal (canal, Voreppe).

La présence de l'eau se signale aussi par la mention d'éléments de la vie de tous les jours :

3.5. «Puits» : Le Puits (Saint-Julien-de-Ratz); Le Puisat (Montagnole / Saint-Pierre-d'Entremont-Isère); Rue du Puits (Chapareillan).

3.6. «Lavoir»: Le Lavoir (Saint-Joseph-de-Rivière); Ruisseau des Lavoirs (Corbel). Peu de lieux-dits, mais de nombreux odonymes : plusieurs Rue du lavoir (Coublevie); Impasse du Lavoir (Saint-Christophesur-Guiers); Rue du Lavoir de Criel (Voiron).

3.7. «Abreuvoir / bachat» : peu de références : Chemin Abreuvoir (SaintHilaire). L'abreuvoir est désigné par «bachat» en de nombreux points de Chartreuse; aux deux points d'enquête de Chartreuse, l'ALJA (carte $\mathrm{n}^{\circ} 640$ «abreuvoir») indique [ba'fa] à Miribel-les-Échelles et [ba'fje] à La Ruchère. Les Bachats (Vimines) est un lieu-dit au bord du nant de 
la Quétille. Cependant l'utilisation de «bachat» peut être motivée par la forme du lieu et non par la présence de l'eau.

3.8. «Vivier» : Le Grand Vivier (Saint-Aupre); Le Vivier (Saint-Étiennede-Crossey); Rue du Vivier (Le Touvet); Chemin du Vivier (Bernin).

3.9. «Citerne» : Citerne (Saint-Julien-en-Ratz).

\section{Signalement indirect :}

4. Végétaux.

La mention de certains végétaux est une attestation incontestable de la présence d'une couverture végétale, aujourd'hui quelquefois disparue. En tant que microtoponymes, ils sont très nombreux et seuls quelques exemples sont cités ci-dessous.

4.1. Aulne : sous sa forme régionale verne (<gaul. verna) : Les Vernes (Entremont-le-Vieux). Les formes suffixées sont majoritaires : Le Vernet (Voissant); Vernat (Les Marches); Vernay (Saint-Cassin); Au Verney (Corenc); Les Vernays (Saint-Aupre); Le Verney (Saint-Pierre-de-Genebroz); Chemin du Verney (Miribel-les-Échelles); Le Verney, Le Verneray (Saint-Pierre-d'Entremont-Isère); Le Verneray (Saint-Pierred'Entremont-Savoie).

4.2. Saule (du lat. SALICEm) : La Saulce (Saint-Pierre-de-Chartreuse). Relevées sur la carte ALJA n ${ }^{\circ} 531$ «saule», les formes dialectales ['sozo / 'soza / 'sodzo] sont régulièrement transformées en «sauge». La Sauge (Saint-Laurent-du-Pont / Saint-Christophe / Entremont-leVieux / Corbel / Saint-Pierre-d'Entremont-Savoie). On rencontre des formes suffixées : Saugey (Saint-Franc), Le Sauget (Aiguebelette, à la limite du massif). Le ruisseau qui traverse le hameau de Saint-Christophe-sur-Guiers se nomme «ruisseau du Sauget». Quelques odonymes: Impasse des Saules (Crolles); rue des Saules (Coublevie).

4.3. Chanvre (du lat. CAnNabe) : Le Chenevas (Miribel-les-Échelles); Le Grand Chenevey (Saint-Pierre-d'Entremont-Savoie); Chenevières (Saint-Étienne-de-Crossey); Au Chenevier / Chenevière (Entremontle-Vieux); Chanevari (Saint-Joseph-de-Rivière); Chenevarie (SaintLaurent-du-Pont); La Combe du Chenevier (Attignat-Oncin).

Osier : il s'agit d'une motivation beaucoup moins productive que les précédentes : Osiers (Coublevie). 


\section{Animaux.}

Les animaux familiers de l'eau sont peu représentés en toponymie : seulement le castor, absent des toponymes de Chartreuse, et la grenouille, souvent par l'intermédiaire d'un nom d'auberge. Une recherche approfondie pourrait indiquer si notre unique relevé La Grenouille (Chapareillan) se range dans cette catégorie.

6. Éléments liés aux activités nécessitant la présence de l'eau.

Lieux d'extraction :

6.1. Sablière : Les Sablières (Saint-Vincent-de-Mercuze); L'Arenier (Saint-Franc), Larrenier (Saint-Joseph-de-Rivière) (du lat. ARENA 'sable' + suffixe <-ARIU); Larinier (Saint-Pierre-de-Chartreuse). Les formes L'Araignée à Attignat-Oncin et Saint-Franc présentent une plaisante remotivation. L'Aragnat (Quaix), même origine mais avec un suffixe diminutif issu de -OTTU.

À noter que toutes les «Sablière» ne sont pas des témoins de la présence de l'eau.

Lieux de transformation :

Les lieux de transformation sont nombreux, d'une densité toutefois différente : la présence d'une forge est plus courante que celle d'une tuilerie par exemple, ce qui se traduit à l'échelle toponymique.

6.2. Forge (< lat. FABRICA) : Les Faverges (Entremont-le-Vieux); Chemin de la Faverge (Saint-Étienne-de-Crossey); La Faurie (Le Sappey).

La forge peut aussi être désignée par l'outil au centre de l'activité : Le Martinet (Saint-Julien-de-Ratz), ou le nom du métier : Le Maréchal (La Bauche); Les Maréchaux (Saint-Ismier).

6.3. Moulin : Le Moulin / Dessous-le-Mollin (Les Échelles); Le Moulin (La Bauche); Moulin (Saint-Étienne-de-Crossey); Le Moulin (SaintAupre / Miribel); Moulin Berger / Moulin Blanc (Vimines); Les Moulins (Saint-Christophe / Attignat-Oncin / La Bauche). Les Chemin du Moulin sont fréquents (Saint-Christophe-sur-Guiers, par exemple).

6.4. Verrerie : La Verrerie (Le Fontanil).

6.5. Tannerie : Rue de la Tannerie (Le Fontanil).

6.6. Tuilerie : La Tiollière (Saint-Cassin) notée «Thiollière» dans le cadastre de 1728; La Tuilerie (Saint-Laurent / Saint-Joseph-de-Rivière); La Tivollière (Coublevie); La Thiolière (Cognin). 
6.7. Poterie : La Poterie (Saint-Thibaud-de-Couz); Impasse Poterie (La Flachère).

6.8. Routoir : Bien que la culture du chanvre soit une activité attestée en Chartreuse $^{2}$, peu de toponymes gardent la trace des lieux de son rouissage; peut-être parce qu'il s'effectuait à l'endroit même de la culture.

Nais (du latin *nasiare FEW 7, 24 2) : Nais (Saint-Franc); Le Né (Saint-Pierre-de-Genebroz - hameau et ruisseau); Fontaine du Nays (Quaix); Le Neyzet (Miribel-les-Échelles) et Chemin du Nezet (qui mène au Neyzet); Pilot de Thorey relève, pour Miribel-les-Echelles, Le Ned attesté au XVIII ${ }^{\mathrm{e}}$ siècle sous la forme Au Naiz.

Nombreuses bases habituelles de l'hydrotoponymie sont absentes de Chartreuse quoique présentes dans le reste de l'Isère; c'est le cas de narce 'terrain marécageux' ou chambon 'terrain au bord de l'eau', par exemple; l'absence de continuateurs du latin CONFLUENTE et du gaulois condat dans la désignation du confluent a déjà été mentionnée.

Il n'en demeure pas moins que les toponymes et microtoponymes liés à l'eau offrent, en Chartreuse, une grande richesse et une grande variété. Sur le territoire de plusieurs communes (Entremont-le-Vieux, Le Fontanil, Les Marches, Miribel-les-Échelles, Saint-Étienne-de-Crossey, Saint-Cassin), on rencontre dix microtoponymes ou plus, qui font référence à l'eau. Ce survol rapide confirme l'intérêt, annoncé en introduction, d'une étude en profondeur sur le sujet.

\section{RÉFÉRENCES BIBLIOGRAPHIQUES}

Atlas

GiLliéron Jules et Edmont Edmond, 1902-1910, Atlas linguistique de la France $(A L F)$, Paris, Champion.

Martin Jean-Baptiste et Tuaillon Gaston, 1971-1978, Atlas linguistique et ethnographique du Jura et des Alpes du Nord (ALJA), Paris, CNRS.

\section{Cadastres}

Cadastres en ligne : <www.cadastre.gouv.fr>.

Mappe sarde : <www.savoie-archives.fr $>$.

2. Fin XIX ${ }^{\mathrm{e}}$ siècle, dans La vie d'Henri Brulard, Stendhal, décrit «d'infâmes routoirs » aux environs de Saint-Joseph-de-Rivière. 
IGN 3333 OT Massif de la Chartreuse nord.

IGN 3334 OT Massif de la Chartreuse sud.

Annuaire téléphonique de l'Isère 2012-2013.

RIVOLI (Répertoire informatisé des voies et lieux-dits), aujourd'hui FANTOIR.

\section{Dictionnaires topographiques}

Pilot de Thorey Emmanuel, 1921, Dictionnaire topographique du département de l'Isère, Romans, imprimerie Jeanne d'Arc.

Vernier Jules-Joseph, 1897, Dictionnaire topographique du département de la Savoie, Chambéry, Imprimerie savoisienne.

\section{Ouvrages}

Bessat Hubert et Germi Claudette, 2001, Les noms du paysage alpin. Atlas toponymique : Savoie, Vallée d'Aoste, Dauphiné, Provence, Grenoble, ELLUG.

Bloch Oscar et Wartburg Walther von, 1975, Dictionnaire étymologique de la langue française, Paris, P.U.F.

Bouvier Jean-Claude, 2002, Les noms de lieux du Dauphiné, Paris, Bonneton.

Cherpillod André, 1986, Dictionnaire étymologique des noms géographiques, Paris, Masson.

Dauzat Albert, Deslandes Gaston et Rostaing Charles, 1982, Dictionnaire étymologique des noms de rivières et de montagnes en France, Paris, Klincksieck.

Delamarre Xavier, 2001, Dictionnaire de la langue gauloise, Paris, Errance.

Gendron Stéphane, 2003, L'origine des noms de lieux en France, Paris, Errance.

Gros Adolphe, 1935, Dictionnaire étymologique des noms de lieu de la Savoie [réédition La Fontaine de Siloé, 1994].

GuEx Jules, 1946, La montagne et ses noms. Études de toponymie alpine, Lausanne, F. Rouge [Martigny, éditions Pillet, 1976].

Lebel Paul, 1956, Principes et méthodes d'hydronymie française, Travaux de la Faculté des sciences, Paris, Les Belles Lettres.

NÈGRe Ernest, 1991, Toponymie générale de la France, Genève, Droz.

PÉGORIER André, 1997, Les noms de lieux en France : glossaire des termes dialectaux, Paris, IGN. 